\title{
Automated Methods in Chiral Perturbation Theory on the Lattice
}

\section{Buḡra Borasoy ${ }^{a}$, Georg M. von Hippel ${ }^{* b}$, Hermann Krebs ${ }^{a}$ and Randy Lewis ${ }^{b}$}

${ }^{a}$ Helmholtz-Institut für Strahlen- und Kernphysik (Theorie), Universität Bonn, Bonn, Germany

${ }^{b}$ Department of Physics, University of Regina, Regina, Saskatchewan, Canada

E-mail: vonhippg@uregina.ca

We present a method to automatically derive the Feynman rules for mesonic chiral perturbation theory with a lattice regulator. The Feynman rules can be output both in a human-readable format and in a form suitable for an automated numerical evaluation of lattice Feynman diagrams. The automated method significantly simplifies working with improved or extended actions. Some applications to the study of finite-volume effects will be presented.

XXIIIrd International Symposium on Lattice Field Theory

25-30 July 2005

Trinity College, Dublin, Ireland

${ }^{*}$ Speaker. 


\section{Introduction}

\subsection{Chiral Perturbation Theory on the Lattice}

One of the most important uses of Chiral Perturbation Theory $(\chi \mathrm{PT})$ is the extrapolation of results from Lattice QCD simulations. Finite volume effects, discretization errors and quark mass extrapolations can all be addressed using $\chi \mathrm{PT}$. In this context, $\chi \mathrm{PT}$ can be regulated with either a continuum formalism [1] or a lattice formalism [2]. Though the lattice approach typically leads to more complicated algebra, it has the advantage of being directly amenable to numerical methods. A recent study of volume dependences in 1-loop lattice $\chi$ PT demonstrates how simple numerical methods are able to replace extensive algebra [3]. Besides its relevance to QCD simulations, lattice $\chi \mathrm{PT}$ is vital for nuclear simulations with chiral effective theories [4].

In lattice regularized $\chi \mathrm{PT}$, the meson fields live on the lattice sites, and gauge fields as usual on the links. Derivatives in the Lagrangian are replaced by appropriately covariantised finite differences. Lattice Chiral Perturbation Theory has been used to compute baryon magnetic moments [5] and to simulate neutron matter [4].

Since Feynman rules become much more complicated on the lattice due to the appearance of trigonometric functions and the loss of Lorentz symmetry, deriving them manually becomes an extremely tedious task, making an automatic procedure desirable.

\subsection{Automated Generation of Feynman Rules}

Procedures to automatically derive Feynman rules for lattice gauge theories from their lattice actions have been known since [6]. A new, efficient and adaptable method was presented in [7]. The great flexibility of this latter method makes it possible to adapt it to the case of $\chi$ PT on a lattice without much difficulty by adding support for separate left- and right-vector fields and extending the $\mathrm{SU}(N)$ algebra to include quark mass matrices. The structure of a meson $n$-point vertex with mesons of flavours $a_{i}$ incoming with momenta $p_{i}$ is

$$
\sum_{\sigma \in \mathscr{S}_{n}} \sum_{i} \frac{1}{F \chi_{i}} f_{i} T_{i}^{a_{\sigma(1)} \cdots a_{\sigma\left(r_{i}\right)}} \exp \left(i \sum_{j} p_{\sigma(j)} \cdot v_{i, j}\right)
$$

where each term can be described by an entity $\left(f, \chi, T, x, y,\left\{v_{j}\right\}\right)$, with $F$ the usual $\chi$ PT parameter (pion decay constant in chiral limit), $\chi$ the chiral order and $f$ the amplitude of the term, $T$ a SU(N) trace, and $x, y$ and $v_{j}$ lattice sites. Entities can be multiplied with the multiplication rule

$$
\begin{gathered}
\left(f, \chi, T, x, y,\left\{v_{j}\right\}\right) *\left(f^{\prime}, \chi^{\prime}, T^{\prime}, x^{\prime}, y^{\prime},\left\{v_{j^{\prime}}^{\prime}\right\}\right)= \\
\left(f f^{\prime}, \chi+\chi^{\prime}, T * T^{\prime}, x, y+y^{\prime}-x^{\prime},\left\{v_{j}\right\} \cup\left\{v_{j^{\prime}}^{\prime}+y-x^{\prime}\right\}\right)
\end{gathered}
$$

following from the above representation of the vertex.

Fields can be expressed in terms of basic entities, and arithmetic operations on fields can be turned into operations on the entities in their expansions. Once the field algebra has been implemented, the perturbative expansion of the action expressed in terms of the fields into entities will occur automatically.

The expansion algorithm is wrapped into a PYTHON program called CHIRPY, which performs the expansion of an arbitrary lattice $\chi \mathrm{PT}$ action given as input and produces the Feynman rules 
in a human-readable $\mathrm{LTT}_{\mathrm{E} X}$, compileable Fortran or special machine-readable format (Fig. 2). The relevant flavour, Lorentz and Taylor [8] algebra is provided in a companion Fortran 95 library called CHIRPER. Other output formats (e.g. for analytical evaluation by a computer algebra program) could be added easily as required.

\subsection{Automated Generation of Feynman Diagrams}

There exist a number of well-known packages for the generation of continuum Feynman diagrams, such as FeynArts [9] or QGraph [10]. None of these, however, easily support the vertices and counterterms of arbitrary order that appear in lattice $\chi$ PT.

For this reason, a straightforward implementation of Wick's theorem is implemented as a PyTHON program called MADELine, which creates the Feynman diagrams contributing to a specified $n$-point function at a specified chiral order. The diagrams are output both as LATEX/FeynMF figures and as Fortran functions using the CHIRPY/CHIRPER routines (Fig. 3).

Using the integration routines provided by the CHIRPER library, these functions can be integrated numerically for an almost automated evaluation of the corresponding lattice Feynman diagrams (where internal propagators carry external on-shell momenta, some user intervention will be necessary to assure the choice of the correct integration contour in the complex plane).

\section{Usage Overview}

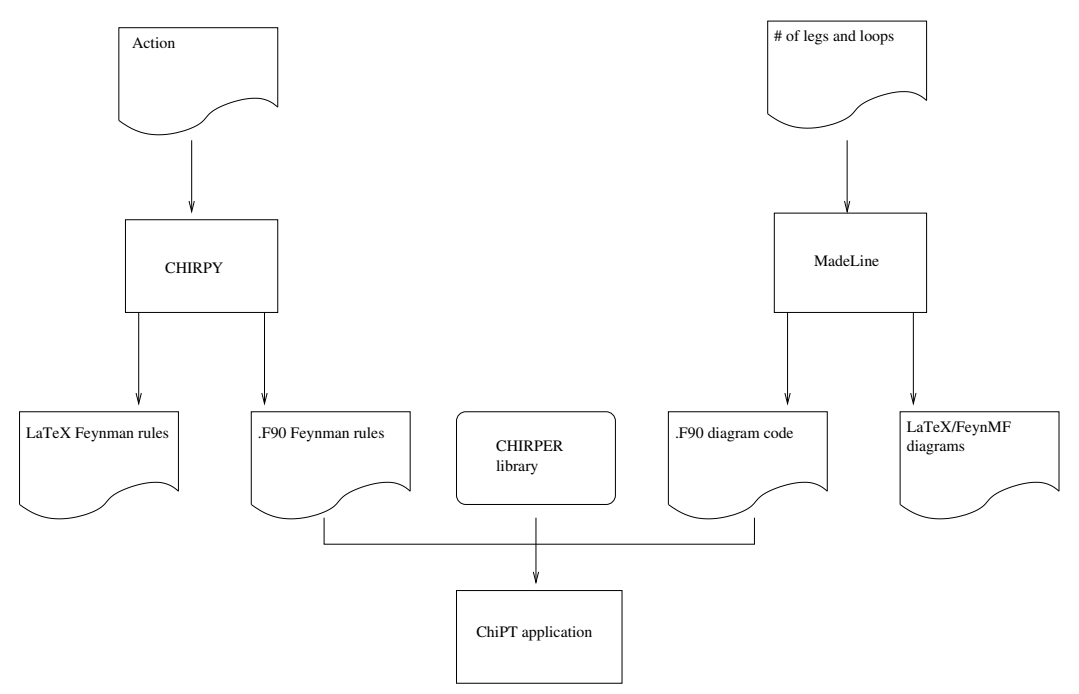

Figure 1: Schematic of usage of the tools

Fig. 1 shows a flow schematic of our tools: The user specifies the action as an input to the CHIRPY main script, which outputs the Feynman rules in the format chosen by the user.

To compute a particular quantity, the user specifies the number of legs and the loop order as input to the MADELINE script, which outputs representations of the relevant Feynman diagrams in both Fortran code and as graphics (where the $\mathrm{LTT}_{\mathrm{E}} \mathrm{X} / \mathrm{FeynMF}$ output may have to be edited by hand to achieve an esthetically pleasing result). 

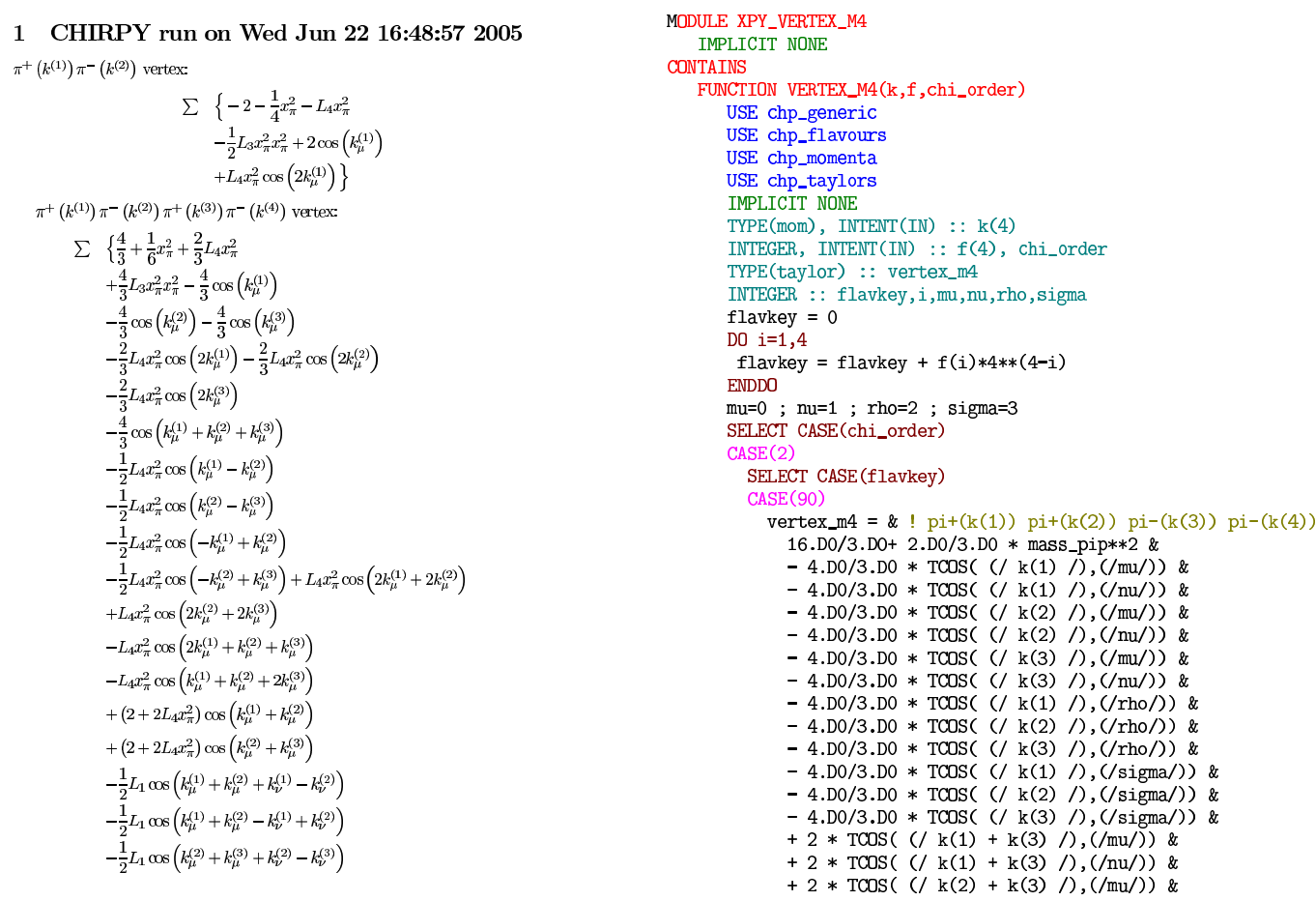

Figure 2: (left) $\mathrm{L}_{\mathrm{E}} \mathrm{X}$ output generated by CHIRPY in human-readable mode. (right) Fortran output generated by CHIRPY in compileable mode. Shown are only the first pages of the multi-page outputs.

The generated code interfaces with the CHIRPER library and the Feynman rules generated by CHIRPY in either compileable or machine-readable format. With just a small amount of userwritten code to bind these components together, a fully functional application of lattice $\chi \mathrm{PT}$ can be produced. Easy-to-use interfaces to initialisation and integration routines are provided by CHIRPER, making the user-written code rather straightforward.

The structure of the interface between CHIRPY and MADELINE allows to reuse the same Feynman rules for a different set of diagrams and vice versa.

\section{An Application to Finite-Volume Effects}

As an example of an application of this framework, we have repeated the one-loop computation of the volume dependence of the pion mass from [3]. The CHIRPER software is so structured that it is easy to evaluate the Feynman diagrams using a variety of summation/integration methods, including VEGAS [11]. This allows to directly extract the difference between the finite-volume and infinite-volume values of the diagrams (instead of using a large, but finite volume to provide a value for the infinite-volume limit, as done in [3]).

The plot of Fig. 4 shows an example of the results which agree well with the earlier evaluation [3] that was based on a hand-coded implementation of manually derived Feynman rules.

The great reduction of the workload in terms of human time by the automated method makes a two-loop calculation appear viable, and a first direct calculation of the two-loop volume dependence of the pion mass is currently under way. 

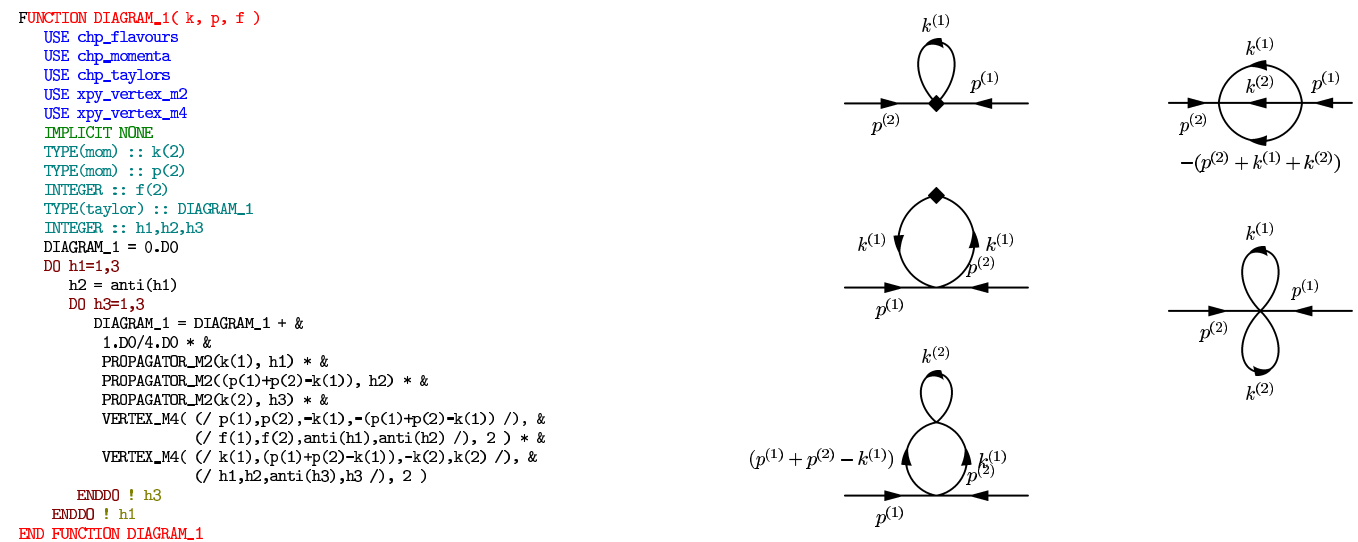

Figure 3: (left) Fortran code generated by MADELINE. (right) FeynMF diagrams generated by MADELINE.

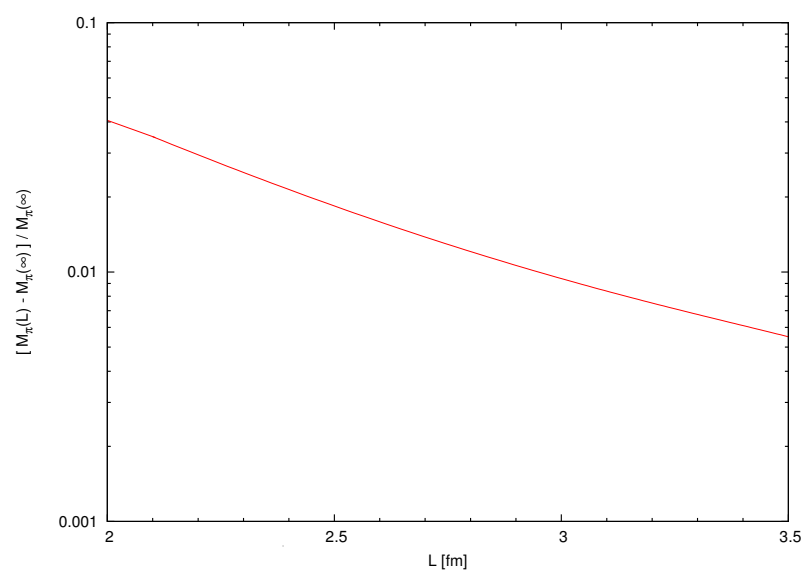

Figure 4: Volume dependence of the pion mass at $M_{\pi}=140 \mathrm{MeV}$

\section{Conclusions}

Chiral Perturbation Theory can be formulated on the lattice, and efficient methods from perturbative Lattice QCD can be translated to the $\chi$ PT case with only moderate effort. A software implementation of an efficient algorithm for generating perturbative expansions exists. The Feynman rules and diagrams can be generated in different formats suitable for use by either humans or computers. An almost automated computation of lattice Feynman diagrams in lattice regularised $\chi \mathrm{PT}$ is possible with these tools.

The automated procedure has successfully been used to repeat finite-volume calculations previously done manually. New finite-volume calculations at the two-loop level using the automated procedure are currently under way. 


\section{Acknowledgements}

GMvH wishes to thank R.R. Horgan and A.G. Hart for useful discussions on automated methods in perturbative Lattice QCD.

This work was supported in part by the Natural Sciences and Engineering Research Council of Canada and by the Government of Saskatchewan.

\section{References}

[1] O. Bär, Chiral perturbation theory at non-zero lattice spacing, Nucl. Phys. Proc. Suppl. 140 (2005) 106-119, [hep-lat/0409123].

[2] R. Lewis and P.-P. A. Ouimet, Lattice regularization for chiral perturbation theory, Phys. Rev. D64 (2001) 034005, [hep-ph/0010043].

[3] B. Borasoy and R. Lewis, Volume dependences from lattice chiral perturbation theory, Phys. Rev. D71 (2005) 014033, [hep-lat/ 0410042 ].

[4] D. Lee, B. Borasoy, and T. Schafer, Nuclear lattice simulations with chiral effective field theory, Phys. Rev. C70 (2004) 014007, [nucl-th/ 0402072 ].

[5] B. Borasoy, R. Lewis, and P.-P. A. Ouimet, Baryon magnetic moments and sigma terms in latticeregularized chiral perturbation theory, Phys. Rev. D65 (2002) 114023, [hep-ph/ 0203199 ].

[6] M. Lüscher and P. Weisz, Efficient numerical techniques for perturbative lattice gauge theory computations, Nucl. Phys. B266 (1986) 309.

[7] A. Hart, G. M. von Hippel, R. R. Horgan, and L. C. Storoni, Automatically generating feynman rules for improved lattice field theories, J. Comput. Phys. 209 (2005) 340-353, [hep-lat/ 0411026 ].

[8] G. M. von Hippel, TaylUR, an arbitrary-order automatic differentiation package for Fortran 95 , physics/0506222.

[9] T. Hahn, Generating Feynman diagrams and amplitudes with FeynArts 3, Comput. Phys. Commun. 140 (2001) 418-431, [hep-ph / 0012260$].$

[10] P. Nogueira, Automatic Feynman graph generation, J. Comput. Phys. 105 (1993) 279-289.

[11] G. P. Lepage, A new algorithm for adaptive multidimensional integration, J. Comput. Phys. 27 (1978) 192. 